\title{
Culture, Caregiving, and Health: Exploring the Influence of Culture on Family Caregiver Experiences
}

\author{
Jennifer R. Pharr,, Carolee Dodge Francis, ${ }^{1}$ Christine Terry, ${ }^{2}$ and Michele C. Clark ${ }^{1}$ \\ ${ }^{1}$ University of Nevada, Las Vegas, NV, USA \\ ${ }^{2}$ Nevada Senior Services, Las Vegas, NV, USA \\ Correspondence should be addressed to Jennifer R. Pharr; jennifer.pharr@unlv.edu
}

Received 13 January 2014; Accepted 17 February 2014; Published 26 March 2014

Academic Editors: W. B. Hansen and K. M. Rospenda

Copyright ( 2014 Jennifer R. Pharr et al. This is an open access article distributed under the Creative Commons Attribution License, which permits unrestricted use, distribution, and reproduction in any medium, provided the original work is properly cited.

\begin{abstract}
Ethnic minorities are expected to experience a greater demand for family caregiving than non-Latino Whites due to their projected population growth. Although the consensus of researchers on caregiving and culture finds that the caregiving experience differs significantly among cultural/ethnic groups, the question remains as to how cultural values and norms influence the caregiver experiences. We conducted an interpretative, phenomenological qualitative analysis of focus group transcripts from four groups (African American, Asian American, Hispanic American, and European American) for cultural influences on caregiving. Data were collected in Nevada between December 7, 2009, and August 20, 2010. Thirty-five caregivers participated in this study. We found commonalities among all of the cultural/ethnic groups in their experiences of the difficulties of caregiving. However, there were some significant differences in the cultural values and norms that shaped the caregiving experience. We categorized these differences as: (a) cultural embeddedness of caregiving, (b) cultural determinants of caregiving responsibilities or taxonomy of caregiving, and (c) cultural values and norms underlying the decision to provide care. The significance of this study is that it highlights the culturally perceived mandate to provide care in the African, Asian, and Hispanic American cultures.
\end{abstract}

\section{Introduction}

Research has shown a link between the stress of caregiving and poor physical/mental health outcomes [1-8]. These links are concerning due to the current prevalence of family caregivers in the USA and the escalating demand for long-term care. The increasing demand can be attributed to several key factors: growth of the older population, increased longevity, the growing burden of chronic diseases, rising health care costs, and an overburdened formal healthcare system $[9,10]$.

In the coming decades, older ethnic minority populations are expected to experience an even greater demand for family caregiving than non-Latino Whites due to projected population growth in these groups [11]. According to the US Census Bureau, data from the 2010 census indicate that the USA population is becoming more diverse by race, with Asian and Hispanic populations having the fasted rates of growth. Conversely, the non-Hispanic White population is growing the slowest and is projected to contribute less to population growth in the coming decades $[12,13]$.

Minority caregivers are less likely than White caregivers to utilize formal support services [11]. The lower use of support services is consistent with studies that show minority caregivers provide more care than their white counterparts [14]. These factors place minorities at a particularly high risk for adverse health effects associated with family caregiving.

Chronic stress due to caregiving has been linked to poor health outcomes, morbidity, and mortality [15]. Preventing and/or ameliorating caregiver stress is vital to sustaining family caregivers. Evidence based car egiver intervention programs have demonstrated positive results for improving caregiver well-being and reducing stress [16]. However, there is a dearth of interventions that report cultural tailoring [11].

Although the consensus of the research on caregiving and culture to date finds that caregiving experiences differ significantly among ethnic groups, the question remains as to how 
culture impacts caregiving $[11,17,18]$. Building upon recommendations in the literature, the overall goal of our analysis was to expand knowledge regarding cultural differences in family caregiving experiences as it relates to perceived expectations of caregiving. More specifically, the research question for this analysis was how do cultural values and norms influence the caregiver experiences?

\section{Methods}

2.1. Research Design. Qualitative research is well suited for understanding phenomena within their context, uncovering links among concepts and behaviors, and generating and refining theory $[19,20]$. Recent literature supports that cultural values are complex and cannot necessarily be defined or explained by a single paradigm or construct, such as individualism and collectivism [21]. Because of this, quantitative measures, such as the use of burden scales, are not sufficient in understanding the complex phenomena of cultures' influence on caregiving. Qualitative methods provide opportunity for a more nuanced search of specific cultural values and their complex meanings. We anticipated that our qualitative analysis would reveal different caregiving experiences among the different ethnic focus groups and provide further insight regarding the influence of culture on caregiving processes.

More specifically, we employed an interpretative, phenomenological research design. A phenomenological study describes the meaning of several individuals' lived experiences of a concept or phenomenon [19]. We utilized focus group data to uncover and illuminate the caregiving experiences of participants from different ethnic groups. The purpose was to reduce individual experiences with caregiving to a description of the universal essence (a grasp of the very nature of the thing) of caregiving as experienced within and between focus groups [19].

2.2. Data Collection. A cross-sectional, semistructured focus group design had been utilized with focus groups of African American, Asian American, Hispanic American, and European American caregivers. Caregivers were recruited who had provided at least 10 hours a week of uncompensated care to an elderly person 60 years of age or older for at least 6 months. Recruitment of caregivers took place in locations where the researchers had good community relationships such as senior center and geriatric care facilities. Additionally, participant recruitment efforts included public service announcements, online and paper participant invitations, and recruitment advertising in local community papers. Data were collected in Las Vegas, NV, between December 7, 2009, and August 20, 2010. IRB approval was obtained prior to data collection. All of the focus groups were recorded and transcripts for each focus group were typed exactly as recorded. Original questions asked of the focus groups were as follows.

(1) Did you grow up with an elder being taken care of by your family or did a neighbor you knew take care of an elder?
(2) What are the common stresses in giving care to your elder?

(3) How do you cope?

2.3. Qualitative Analysis. Two researchers analyzed the raw transcript data. The data consisted of three focus group transcripts received from African American focus groups and two transcripts received from each of the following focus groups: Asian American, Hispanic American, and European American caregivers. We analyzed the transcripts and evaluated the data for cultural influences on caregiving.

We utilized The Updated Sociocultural Stress and Coping Model to guide our data analysis [18]. Two researchers from the team analyzed the focus group transcripts using the following steps. (a) In the initial step of the analysis, we read each transcript individually several times while looking for and flagging emerging patterns. (b) The second step, we noted the initial and global impressions of each transcript. (c) The third step, we compared transcripts to each other in a side-byside analysis of all of the focus group transcripts. Common themes pertaining cultural values and norms were sought as these are the variables in the Sociocultural Stress and Coping Model which are posited to vary among ethnic/cultural groups. (d) In the fourth step of the analysis, we reread and coded each focus group transcript separately. Themes were identified and coded using colored highlighters. Lastly, we categorized narratives illustrating the common themes for each focus group [20].

2.4. Validity and Reliability. "In phenomenological perspective, the meanings of truth, validity, and reliability are used differently from the conventional terms as they are applied in quantitative research. Verification and content of the data require a new approach" [22]. Throughout the data analysis, themes were always verified by two of our experienced qualitative researchers. They coded transcripts separately to encompass a wider analytical net and achieved a base agreement percentage of $90 \%$. This is one of Osborne's four strategies for assessing the validity of qualitative data [23]. We reviewed the categorization of data and meanings into themes to verify consistency of the clustering. This was instrumental for us to determine common themes that were woven throughout the focus group transcripts.

\section{Findings}

3.1. Participant Demographics. A total of 35 caregivers participated in the study: 9 European American, 7 Asian American, 8 Hispanic American, and 11 African American. There were 32 female and 3 male participants. The ages of the participants ranged from 35 to 83 years, with a mean age of 52.6 years. The breakdown of caregivers' ages and care receivers' ages is provided in Table 1.

The sample was highly educated, with 54\% achieving a college degree and 26\% achieving a high school diploma. Asian Americans were the most highly educated group with $85.7 \%$ achieving a college degree, followed by European Americans with $66.7 \%$ receiving a college education. 
TABLE 1: Age of caregivers and care receivers.

\begin{tabular}{lcccc}
\hline & $\begin{array}{c}\text { Caregiver } \\
\text { range }\end{array}$ & $\begin{array}{c}\text { Caregiver } \\
\text { mean }\end{array}$ & $\begin{array}{c}\text { Care receiver } \\
\text { range }\end{array}$ & $\begin{array}{c}\text { Care receiver } \\
\text { mean }\end{array}$ \\
\hline $\begin{array}{l}\text { European } \\
\text { American }\end{array}$ & $61-83$ & 65.2 & $62-89$ & 81.6 \\
$\begin{array}{l}\text { African } \\
\text { American }\end{array}$ & $41-74$ & 54.9 & $66-85$ & 75.6 \\
$\begin{array}{l}\text { Asian } \\
\text { American }\end{array}$ & $38-52$ & 43.8 & $72-87$ & 80.4 \\
$\begin{array}{l}\text { Hispanic } \\
\text { American }\end{array}$ & $26-56$ & 46.6 & $72-87$ & 77.37 \\
\hline
\end{tabular}

The Hispanic American and African American groups were very similar in terms of college education with $37.5 \%$ of Hispanic Americans and 36.4\% of African Americans achieving a college education. Unlike the European American and Asian American groups, more Hispanic Americans (12.5\%) and African Americans (27.3\%) attended technical or trade schools.

3.2. Qualitative Results. We used The Updated Sociocultural Stress and Coping Model (SCSCM) for Culturally Diverse Family Caregivers as the basis for analysis for all four focus groups' qualitative data [18]. We found some significant differences in the cultural values and norms that shaped the caregiving experience. These differences were captured in the three categories, all pertaining to the Cultural Values construct of the Updated SCSCM: (a) cultural embeddedness of caregiving, (b) cultural determinants of caregiving responsibilities or taxonomy of caregiving, and (c) cultural values and norms underlying the decision to provide care. For each focus group, we found unique themes pertaining to each of the three categories which highlighted cultural differences in the caregiving experience.

\subsection{Cultural Values and Norms}

3.3.1. Theme 1 Cultural Embeddedness of Caregiving: "It Is Not Questioned". According to Brislin, "culture refers to widely shared ideals, values, formation, and uses of categories, assumptions about life, and goal-directed activities that become unconsciously or subconsciously accepted as right and correct by people who identify themselves as members of a society" [24]. Caregiving was seen as "right and correct" by all of the focus groups. However, for some of the groups, caregiving was an expected part of life that was passed down from generation to generation. In fact, caregiving was so embedded in the life experience for some of the groups that the decision to care or not to care was irrelevant; caregiving was just something that was done without question. To provide care for one's family or community was deeply rooted in the cultural subconsciousness, arising "naturally" without conscious thought. This perspective was shared by Asian American, Hispanic American, and African American focus group participants.
Asian American, Hispanic American, and African American participants reported seeing multiple examples of caregiving, not just within their own families but throughout their communities. In fact, it was so culturally ingrained to care for one's family that, when the time came, care was provided without question. For Asian Americans, caregiving was described as just a normal thing to do. One Asian American caregiver explained:
"Like, don't you feel like sometimes it's just in- grained in us, so much as a culture because we saw it as a kid. We saw our parents take care of their parents, and when you were growing up, there was always an elder person in the home. You just saw that all the time, you saw it in the community, you saw it in your country." (FG-4-ASA number 3).

Hispanic Americans portrayed caregiving as an instrumental and defining aspect of the family experience, so much so, that not only were other alternatives for formal care outside of the family not considered, they also were not even known to exist:

"I mean, that's taboo for us but also you feel that way so it doesn't even come to your head. It's just, I mean, you're just so close with your family, that family's family, and you take care of each other regardless." (F5 H/L number 2).

African Americans depicted caregiving as a part of cultural/ family history that has been passed on for generations. Therefore, caregiving came "naturally" and was "nothing new":

"You know, it just come naturally, that's been in our family a long time we have had a lot of caregivers. A lot of folks waited on people for a number of years took over the role and so you know it's nothing new." (F9 AfAm number 4).

In contrast to the cultural embeddedness of caregiving that was illuminated in the Asian American, Hispanic American, and the African American caregiving experiences, the European American caregivers expressed that they had no direct examples of caregiving growing up. In some cases, participants stated that they had been aware of family members caring for family members but never observed it directly.

3.3.2. Theme 2 Cultural Determinants of Caregiving Responsibilities or Taxonomy of Caregiving. Positional Role. A culture's orientation toward individualism and collectivism and the degree to which the values of these orientations are embodied have also been shown to influence the role of caregiving, specifically who is responsible to care [25-28]. In some cultures, particularly those with a collectivistic orientation, the role of caregiving is culturally prescribed based on a set hierarchy of "who" is supposed to care and/or "traditional" gender ideology that assigns care work to women [27].

In our study, Asian American caregivers emphasized that one reason they were providing care was that it was their position to take care of the elderly. In the following text, an Asian American focus group participant discussed 
the expectation of the daughter and or daughter-in-law's "positional role" to provide care regardless of competing role responsibilities. The onus is on women not men to provide care:

"You just saw that the daughter or the daughter-
in-law took care of the mother, it wasn't the son,
it was the daughter or the daughter-in-law that
would go." (FG-4-ASA number 1).

The following example describes how the "positional role" to care for a parent was nurtured in the family, beginning at a young age. Role expectations were determined based on a child's gender and birth order in the family and shaped the traits and activities that were encouraged and those that were discouraged:

"I think as a culture for us, the youngest one is expected [to care for elders]. The older son is expected to succeed actually, and if, you know, something happens to the parents, he is expected to take care of the siblings. .... I was never encouraged to go to college where my brothers and sister were.... Yeah, they expected me to get married and then to take care of them.... They trained me to entertain, to be a good wife, to marry someone successful but they never wanted me to really to have a real career." (FG-4-ASA number 2).

In other collectivistic cultures, we also saw a strong cultural prescription for caregiving. In Hispanic/Latino cultures, caregiving was primarily prescribed based on female gender but does not appear to be governed by rules which mandate a strict hierarchy designating specific female family members. There were clearly delineated gender expectations among Hispanic caregivers.

The Hispanic American focus group participants described caregiving as a female duty that was often assumed by the oldest daughter. The following passage describes how this role was nurtured from a young age, whereby the oldest daughter was expected to care for her younger siblings growing up and then for elders in adulthood:

"Because I'm the oldest, since I was little, when my parents would go to work I was in charge of the household. I'm the oldest out of 5, so since I was little that's always been part of my life, my role to take care of my younger siblings. So as I got older I kept that role and even now with my grandmother it's like, well, if I could do it with my siblings, I could do it with my grandmother." (F5 $H / L$ number 2).

For European and African American caregivers, female gender also prescribed caregiving. However, for European Americans caregiving did not appear to be culturally prescribed, as there was no cultural explanation provided in any of the focus group transcripts. Rather personal responsibility not culture was what influenced the "decision to provide care." The decision to provide care was what sets the European
American caregivers apart from the other focus groups in terms of the caregiving role and demonstrates the powerful influence of culture on the caregiving experience. For all of the focus groups, with the exception of the European American group, caregiving was so culturally embedded and prescribed that it was accepted and enacted without question, therefore making the decision to provide care a moot point; there was no need for a decision when it had already been decided.

Family Kinship. For the African American caregivers, caregiving responsibilities extended beyond immediate family members and encompass kinship relationships. Close friends and neighbors were considered to be part of one's family and were cared for as such. A caregiver described taking care of her friend's mother as if she were her own mother. She actually referred to her friend as her "sister" and her friend's mother as her "mother."

3.3.3. Theme 3 Cultural Values and Norms Underlying the Decision to Provide Care: "Cannot Say No". Maintaining harmonious relationships with others is paramount in collectivistic cultures [29]. In this study, saying "no" to someone was viewed as rejection and abandonment, the ultimate affront to social harmony, and was deemed unacceptable by collectivistic cultures. For Asian American focus group participants, saying "no" posed far too great a risk to family relationships:

\section{"The family ties are so important and I don't want to make waves for me, for our family.... If you're the one designated as the caregiver and if you just flat out say "no, I'm not doing it anymore" it would cause so much family upset that it wouldn't be worth to me to say flat out "I am not doing this anymore, you guys do it" because, you know, it would just be too much, too much to upset the cart apple cart. I mean, it just it doesn't work for my family to not get along, and so you don't say no." (FG-4-ASA number 1).}

Preserving social/family harmony was a cultural imperative that was not worth risking. Saying no to caregiving responsibilities does not only threaten family harmony but it would also undermines the central tenants of filial piety: respect and loving one's parents, not bringing any type of dishonor to one's parents, taking good care of one's parents, and obeying one's parents [30]. Filial piety mandates more than just responsibility for care; it requires that elders are cared for in the way that they want to be cared for and that care requests are respected and enacted. The consequences for not doing so manifested in guilt and bad feelings about oneself, which was echoed by several Asian American caregivers. One caregiver stated

"So, that's it. You feel, just feeling guilty because you feel like you are obligated. You know what I'm saying? You feel like obligated that you have to do these things for her. Because she's family, she's older, she's an elderly. There is nobody, you know, 
you are the only family that she has, so it more that there is the obligation part of it, an obligation." (FG-3-ASA number 2).

While another Asian American caregiver stated

"Because I feel I'm selfish. I am being selfish about myself because I am only thinking of what I want and not considering what the others need." (FG-3ASA number 1).

And a third said

"Oh, I would feel so bad about myself to. Not be able to give into her request or to make adjustments just to make her feel better. I would be I would feel so bad." (FG-3-ASA number 3).

For all of the Asian American caregivers, saying no to the caregiving responsibility was considered culturally unacceptable. This was emphatically attested to throughout the focus group transcripts. When asked, why could not you say no? Asian American caregivers responses were

"It's not acceptable to our culture. Because its, you're supposed to take care of each other. You're supposed to take care of older people. If somebody needs help, you have to help them. If somebody comes to you and wants help, you have to do it and turning them away is not acceptable." (FG-3-ASA number 2).

And

"Yeah, same thing with Koreans. It would be, well first, it would be completely unheard of for child not to take care of the parents. And if it did, if a child did reject the parents and not take care of them, it would be a major catastrophic faux pas. I mean it would, you'd be on the verge of getting stoned." (FG-3-ASA number 3).

Similarly to the Asian American caregivers, Hispanic American and African American caregivers also placed high priority on family relationships. This was depicted by caregivers in the following texts. A Hispanic caregiver said

"And it's the same with us. That was a big no-no. Your family stays with you. You don't send them to away for somebody to take care of them, for them to die alone. They die with you, they stay with you. That's what was always with us." (F5 H/L number 2).

An African American caregiver stated

"Family takes care of family. It used to be, a long time ago, the community came in together to help. But you know, family has always taken care of families. That is a part of our culture." (F5 AfAm number 1).
Conversely, European American caregivers were not culturally bound to provide care to family members, rather caregiving appeared to be influenced more by individual, family, and societal values. Marriage vows: "for better or worse," reciprocity, a personal sense of duty, and the Golden Rule: Do unto others as you would have them do unto you, were all cited as influencing the decision to accept and provide the caregiving role. These values are elucidated in the following passage from a European American caregiver:

"I think over all, our American society, you know, you just don't leave someone cause their sick. You know you leave them if they're not nice or they beat you or they don't work or they drink or whatever. But if their ill, you don't abandon them." (FG-2EA number 2).

\section{Discussion}

We examined the relationship between culture and caregiving. The data supported that cultural values and norms influenced caregiving experiences, specifically cultural embeddedness of caregiving, cultural determinants of caregiving responsibilities or taxonomy of caregiving, and cultural values and norms underlying the decision to provide care.

4.1. Cultural Embeddedness. For Asian American, Hispanic American, and African American focus group participants, we found caregiving was so embedded in the life experience that the decision to care or not to care was irrelevant; caregiving was just something that was done without question. To provide care for one's family or community was deeply rooted in the cultural subconsciousness, arising "naturally" without conscious thought.

In contrast, the European American caregivers expressed that they had no direct examples of caregiving growing up. In some cases, participants stated that they had been aware of family members caring for family members but never observed it directly. Caregiving was not embedded in the life experience of the European American caregivers and was thus unexpected. This finding supports previous literature that describes caregiving as a normative family activity but with culture shaping how much it is considered a normal, expected aspect of life versus an unexpected disruption [31, 32]. Additionally, our finding that caregiving was not embedded in the life experience of European American caregivers supports previous research by Wallhagen and YamamotoMitani that, for American caregivers, "the cultural norm is not specific enough to provide a framework within which to view caregiving as part of one's expected, normal life course" [32].

4.2. Taxonomy of Caregiving. Our finds indicated that there were significant differences among the different focus groups in terms of caregiving taxonomy or who was supposed to provide care. Asian cultures, rooted in Confucian ideology, subscribe to a cultural model that dictates a rigid taxonomy for caregiving based on one's gender and position within the family. This caregiving taxonomy requires daughters, 
specifically daughters-in-laws, to provide ongoing daily assistance to their in-laws, parents, and older relatives [33]. In the event that there is no daughter-in-law or the daughterin-law is unable to provide care, then the caregiving role defers to unmarried daughters, then to married daughters, and lastly to extended female family members, moving along a hierarchy of preference. This taxonomy was further confirmed in Wallhagen and Yamamoto-Mitani's qualitative study of Japanese and American Caregivers [32].

Based on her study of Latina caregivers, Ibarrra posits that women learn about caregiving from their experiences in family groups. Ibarra argues that family caregiving lessons delineate a gendered division of labor [34]. Hispanic American caregivers also described a strong cultural prescription to care for family. However, the caregiving role was assigned based on female gender and was not governed by a strict hierarchy that designates specific female family members.

African American participants, female gender also prescribed caregiving. African American caregivers described a cultural prescription for caregiving that extends the responsibility for care beyond immediate family members to include kin, that is, close friends and neighbors. In their research on effective coping strategies of African Americans, Daly and colleagues discuss how the Afrocentric paradigm has contributed to the survival and success of the African American community [35]. The Afrocentric paradigm "proposes that in African culture humanity is viewed as a collective rather than as individuals and that this collective view is expressed as a shared concern and responsibility for the well-being of others" $[36,37]$. The shared concern and responsibility for the well-being of others were pervasive in the African American focus group transcripts and affirmed in the focus group participants' conceptualization of family and connection to their community.

European American caregivers lacked a cultural prescription for caregiving, as there was no cultural explanation provided in any of the focus group transcripts. Rather personal responsibility not culture was what influenced the decision to provide care. Our findings concur with previous research which posits that a culture's orientation toward individualism and collectivism and the degree to which the values of these orientations are embodied have also been shown to influence the role of caregiving, specifically who is responsible to care [25-28].

\subsection{Cultural Values and Norms Underlying the Decision to} Provide Care. Several studies find that many collectivistic cultures prioritize social harmony over the desire to achieve or strive for personal goals [38-40]. Collectivistic cultures promote forbearance, which entails keeping problems to oneself, and quietly accepting and enduring adversity, as a favorable coping mechanism because it sustains social harmony. The preservation of positive social relations is at the forefront of concern for collectivistic individuals [41].

We found obligation and prioritization of one's family and a strong desire to maintain harmonious relationships mandated and sustained caregiving responsibilities. For Asian American caregivers these values were embodied in the cultural value of filial piety and were manifested in the pervasive belief that it was unacceptable to say no to family and to caregiving responsibility. "Cannot say no" was a cultural imperative that extends beyond family and caregiving relationships and was described as unthinkable and culturally unacceptable by Asian American caregivers.

As cited in Flores et al. "familism refers to the value of the family as an institution, the ideal of interdependence in family relationships, and the priority placed on reliance on family members rather than on more impersonal institutions for instrumental, emotional, and material support" [42]. Familism was manifested for both African American and Hispanic American caregivers in the following cultural imperative: "family takes care of family." To say no to caregiving and to place the responsibility for care in the hands of a stranger and or institutions outside the family violated the values of familism and was seen as turning ones back on family.

4.4. Age of Caregivers. It should also be noted that the European American caregivers were older than the Asian American, Hispanic American, and African American caregivers in our focus groups. It might be reasoned that older caregivers would be more likely to see caregiving as an expected part of life as their partner or spouse ages. However, that was not the case in this study. The younger caregivers in the Asian American, Hispanic American, and African American focus groups were more likely to see caregiving as an expected part of life compared to the older European American caregivers.

4.5. Practice Implications. Our findings provide some important insights into how culture influences caregiving experiences. The degree to which caregiving is culturally embedded and prescribed may preclude some caregivers from questioning the caregiving role and/or seeking support. Therefore, the degree of isolation for some ethnic groups may be even more profound. Early assessment and detection of isolation and stress is critical to sustaining caregivers' health and well-being. This is often challenging in minority groups for various reasons: lack of access to programs and services, language barriers, mistrust of government programs and social service providers, a history of mistreatment and/or a lack of understanding by service practitioners, and cultural values that discourage support seeking outside the family.

Because of this, the way that caregiver programs and services are packaged and presented to different minority groups is essential to their success. For example, caregivers who believe that it is their positional role or duty to provide care and/or do not believe in placing the responsibility for care in the hands of individuals or institutions outside the family, respite care may not be appealing if it is presented as a way to get a break from caregiving responsibilities. However, if instead it is presented as a way to recharge your battery so that you will be able to provide better care to your family member, caregivers may be more apt to consider the service. Caregiver intervention programs should never seek to change cultural values or beliefs; rather they should maximize the strengths of ethnic groups. 
We recognize that there are limitations to this analysis. As with any research, especially qualitative, there is a potential for research bias. We attempted to minimize this bias by having two skilled qualitative researchers code the data. A limitation of qualitative research is the inability to generalize the results and we recognize that as a limitation of this study. Additionally, the sample was predominantly female and may not illustrate a male caregiver's point of view. At times during the interview process, leading questions were asked which may have influenced participants' responses.

\section{Conclusion}

Our findings expand upon the existing literature regarding culture and caregiving and provide further insight into how culture impacts caregiving. We found that cultural values and norms influenced the following: perception of the caregiving role in terms of the role being an expected or unexpected part of the life course; the cultural embeddedness of caregiving also impacted whether or not caregiving was viewed as a choice or an expected duty; taxonomy of caregiving or a prescription for who was supposed to provide care; and lastly the values that familism and filial piety mandated and sustained caregiving responsibilities and strongly prevented caregivers from saying no to caregiving. These findings also underscore the importance of furthering our understanding regarding specific cultural nuances that impact caregiver stress and coping processes as well as support the necessity of qualitative measures to accomplish this.

\section{Conflict of Interests}

The authors declare that there is no conflict of interests regarding the publication of this paper.

\section{Acknowledgments}

This study was funded in part through the URBAN 21 grant from the Urban Sustainability Initiative at the University of Nevada Las Vegas. The grant was awarded to Dr. Michele C. Clark and Dr. Carolee Dodge Francis.

\section{References}

[1] S. C. Ho, A. Chan, J. Woo, P. Chong, and A. Sham, "Impact of caregiving on health and quality of life: a comparative population-based study of caregivers for elderly persons and noncaregivers," Journals of Gerontology A, vol. 64, no. 8, pp. 873879, 2009.

[2] A. C. King, R. K. Oka, and D. R. Young, "Ambulatory blood pressure and heart rate responses to the stress of work and caregiving in older women," Journals of Gerontology, vol. 49, no. 6, pp. M239-M245, 1994.

[3] S. Lee, G. A. Colditz, L. F. Berkman, and I. Kawachi, "Caregiving and risk of coronary heart disease in U.S. women: a prospective study," American Journal of Preventive Medicine, vol. 24, no. 2, pp. 113-119, 2003.

[4] M. Pinquart and S. Sörensen, "Differences between caregivers and noncaregivers in psychological health and physical health: a meta-analysis," Psychology and Aging, vol. 18, no. 2, pp. 250267, 2003.

[5] J. Robison, R. Fortinsky, A. Kleppinger, N. Shugrue, and M. Porter, "A broader view of family caregiving: effects of caregiving and caregiver conditions on depressive symptoms, health, work, and social isolation," Journals of Gerontology B, vol. 64, no. 6, pp. 788-798, 2009.

[6] R. Schulz, J. Newsom, M. Mittelmark, L. Burton, C. Hirsch, and S. Jackson, "Health effects of caregiving: the caregiver health effects study: an ancillary study of the cardiovascular health study," Annals of Behavioral Medicine, vol. 19, no. 2, pp. 110-116, 1997.

[7] R. Schulz and S. R. Beach, "Caregiving as a risk factor for mortality: the caregiver health effects study," Journal of the American Medical Association, vol. 282, no. 23, pp. 2215-2219, 1999.

[8] W. S. Shaw, T. L. Patterson, M. G. Ziegler, J. E. Dimsdale, S. J. Semple, and I. Grant, "Accelerated risk of hypertensive blood pressure recordings among Alzheimer caregivers," Journal of Psychosomatic Research, vol. 46, no. 3, pp. 215-227, 1999.

[9] R. W. Johnson and J. M. Wiener, "A profile of frail older Americans and their caregivers," The Urban Institute, Washington, DC, USA, February 2006, http://www.urban.org/uploadedpdf/ 311284_older_americans.pdf.

[10] T. E. Seeman, S. S. Merkin, E. M. Crimmins, and A. S. Karlamangla, "Disability trends among older Americans: National Health and Nutrition Examination surveys, 1988-1994 and 1999-2004," American Journal of Public Health, vol. 100, no. 1, pp. 100-107, 2010.

[11] A. M. Napoles, L. Chadiha, R. Eversley, and G. MorenoJohn, "Reviews: developing culturally sensitive dementia caregiver interventions: are we there yet?" American Journal of Alzheimer's Disease and other Dementias, vol. 25, no. 5, pp. 389406, 2010.

[12] U.S. Census Bureau, "Census Briefs: Overview of Race and Hispanic Origin: 2010," Issued March 2011, http://www.census .gov/prod/cen2010/briefs/c2010br-02.pdf.

[13] U.S. Census Bureau, National Population Projections, http:// www.census.gov/population/projections/data/national/.

[14] J. J. McCann, L. E. Hebert, L. A. Beckett, M. C. Morris, P. A. Scherr, and D. A. Evans, "Comparison of informal caregiving by black and white older adults in a community population," Journal of the American Geriatrics Society, vol. 48, no. 12, pp. $1612-1617,2000$.

[15] J. P. Gouin, "Chronic stress, immune dysregulation, and health," American Journal of Lifestyle Medicine, 2011, http://ajl.sagepub .com/content/early/2011/01/19/1559827610395467.

[16] A. F. Elliott, L. D. Burgio, and J. Decoster, "Enhancing caregiver health: findings from the resources for enhancing Alzheimer's Caregiver Health II intervention," Journal of the American Geriatrics Society, vol. 58, no. 1, pp. 30-37, 2010.

[17] M. P. Aranda, "The influence of ethnicity and culture on the caregiver stress and coping process: a sociocultural review and analysis," Gerontologist, vol. 37, no. 3, pp. 342-354, 1997.

[18] B. G. Knight and P. Sayegh, "Cultural values and caregiving: the updated sociocultural stress and coping model," Journals of Gerontology B, vol. 65, no. 1, pp. 5-13, 2010.

[19] J. W. Creswell, Qualitative Inquiry \& Research Design: Choosing Among Five Approaches, Sage, Thousand Oaks, Calif, USA, 2nd edition, 2007.

[20] J. Green and N. Thorogood, Qualitative Methods For Health Research, Sage, London, UK, 2004. 
[21] M. H. Segall, W. J. Lonner, and J. W. Berry, "Cross cultural psychology as a scholarly discipline: on the flowering of culture in behavioral research," American Psychologist, vol. 53, no. 10, pp. 1101-1110, 1998.

[22] Y. Takano, "Coping with domestic violence by Japanese Canadian women," in Handbook of Multicultural Perspectives on Stress and Coping, P. T. P. Wong and L. C. J. Wong, Eds., pp. 319360, Springer, New York, NY, USA, 2006.

[23] J. W. Osborne, "Some basic existential-phenomenological research methodology for counsellors," Canadian Journal of Counselling, vol. 24, no. 2, pp. 79-91, 1990.

[24] P. T. P. Wong, L. C. J. Wong, and C. Scott, "Beyond stress and coping: the positive psychology of transformation," in Handbook of Multicultural Perspectives on Stress and Coping, P. T. P. Wong and L. C. J. Wong, Eds., pp. 1-26, Springer+Business Media, New York, NY, USA, 2006.

[25] M. O. Asai and V. A. Kameoka, "The influence of sekentei on family caregiving and underutilization of social services among Japanese caregivers," Social Work, vol. 50, no. 2, pp. 111-118, 2005.

[26] K.-H. Hsueh, J. Hu, and S. Clarke-Ekong, "Acculturation in filial practices among U.S. Chinese caregivers," Qualitative Health Research, vol. 18, no. 6, pp. 775-785, 2008.

[27] K. S. Lee, "Gender, care work, and the complexity of family membership in Japan," Gender and Society, vol. 24, no. 5, pp. 647-671, 2010.

[28] M. I. Wallhagen and N. Yamamoto-Mitani, "The meaning of family caregiving in Japan and the United States: a qualitative comparative study," Journal of Transcultural Nursing, vol. 17, no. 1, pp. 65-73, 2006.

[29] H. R. Markus and S. Kitayama, "Culture and the self: implications for cognition, emotion, and motivation," Psychological Review, vol. 98, no. 2, pp. 224-253, 1991.

[30] Y. T. Dai and M. F. Dimond, "Filial piety. A cross-cultural comparison and its implications for the well-being of older parents," Journal of Gerontological Nursing, vol. 24, no. 3, pp. 13-18, 1998.

[31] M. I. Wallhagen and W. J. Strawbridge, "My parent—not myself: contrasting themes in family care," Journal of Aging and Health, vol. 7, no. 4, pp. 552-572, 1995.

[32] N. Yamamoto and M. I. Wallhagen, "The continuation of family caregiving in Japan," Journal of Health and Social Behavior, vol. 38, no. 2, pp. 164-176, 1997.

[33] R. Ngan and W. Wong, "Injustice in family care of the Chinese elderly in Hong Kong," Journal of Aging \& Social Policy, vol. 7, no. 2, pp. 77-94, 1995.

[34] M. L. Ibarra, "The tender trap: Mexican immigrant women and the ethics of elder care work," Aztlan, vol. 28, no. 2, pp. 87-113, 2003.

[35] A. Daly, J. Jennings, J. O. Beckett, and B. R. Leashore, "Effective coping strategies of African Americans," Social Work, vol. 40, no. 2, pp. 240-248, 1995.

[36] N. Ak'bar, "Afrocentric social services for human liberation," Journal of Black Studies, vol. 14, pp. 395-413, 1984.

[37] L. N. Houston, Psychological Principles and the Black Experience, University Press of America, New York, NY, USA, 1990.

[38] A. Ben-Ari and Y. Lavee, "Cultural orientation, ethic affiliation, and negative daily occurrences: a multidimensional crosscultural analysis," American Journal of Orthopsychiatry, vol. 74, no. 2, pp. 102-111, 2004.

[39] C. A. McCarty, J. R. Weisz, K. Wanitromanee et al., "Culture, coping, and context: primary and secondary control among Thai and American youth," Journal of Child Psychology and
Psychiatry and Allied Disciplines, vol. 40, no. 5, pp. 809-818, 1999.

[40] S. Arzu Wasti and L. M. Cortina, "Coping in context: sociocultural determinants of responses to sexual harassment," Journal of Personality and Social Psychology, vol. 83, no. 2, pp. 394-405, 2002.

[41] C. Yeh, A. Kwong Arora, and A. Wu, "A new theoretical model of collectivistic coping," in Handbook of Multicultural Perspectives on Stress and Coping, P. T. P. Wong and L. C. J. Wong, Eds., pp. 56-72, Springer+Business Media, New York, NY, USA, 2006.

[42] Y. G. Flores, L. Hinton, J. C. Barker, C. E. Franz, and A. Velasquez, "Beyond familism: a case study of the ethics of care of a latina caregiver of an elderly parent with dementia," Health Care for Women International, vol. 30, no. 12, pp. 1055-1072, 2009. 


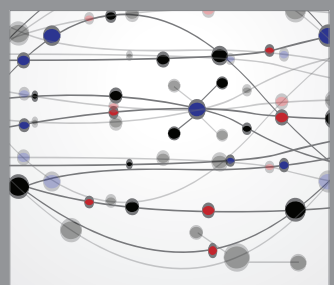

The Scientific World Journal
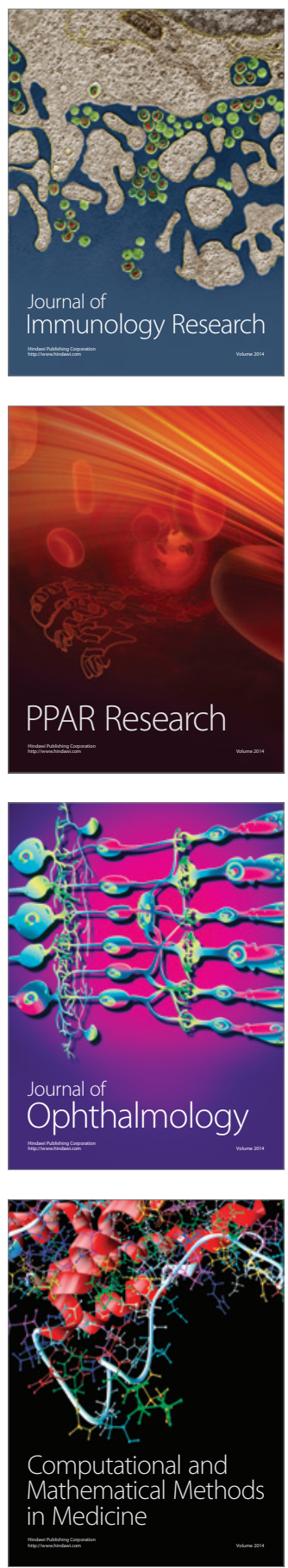

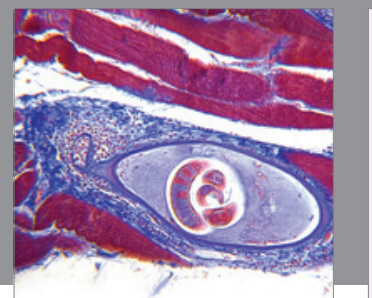

Gastroenterology

Research and Practice
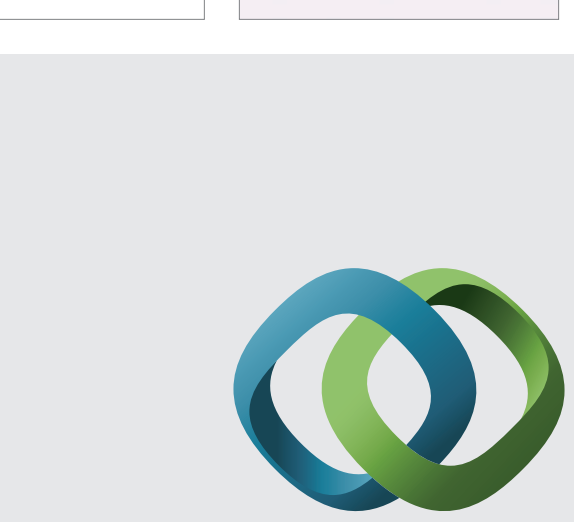

\section{Hindawi}

Submit your manuscripts at

http://www.hindawi.com
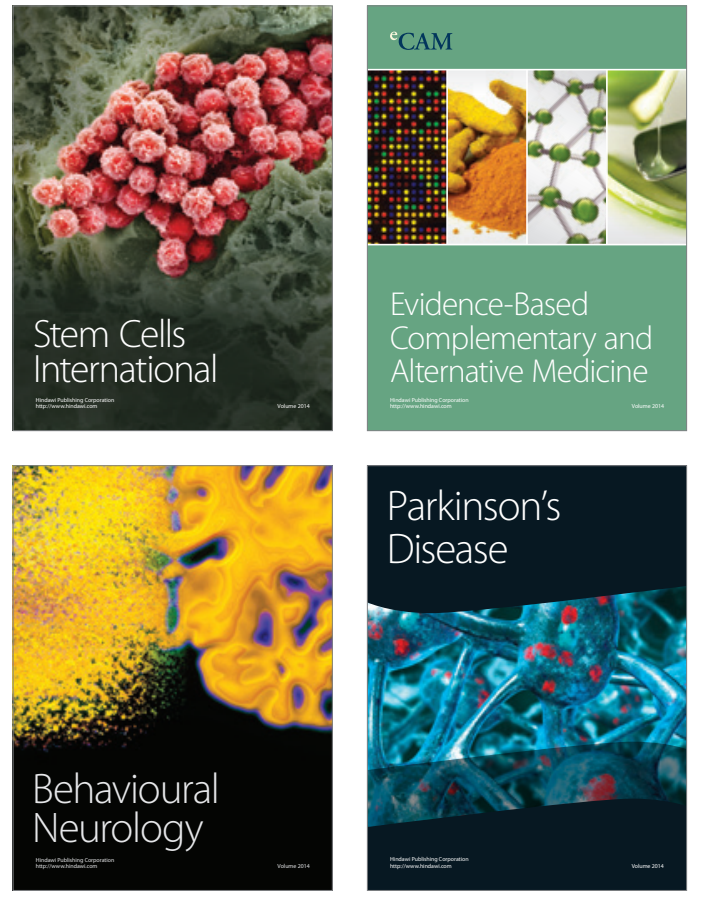
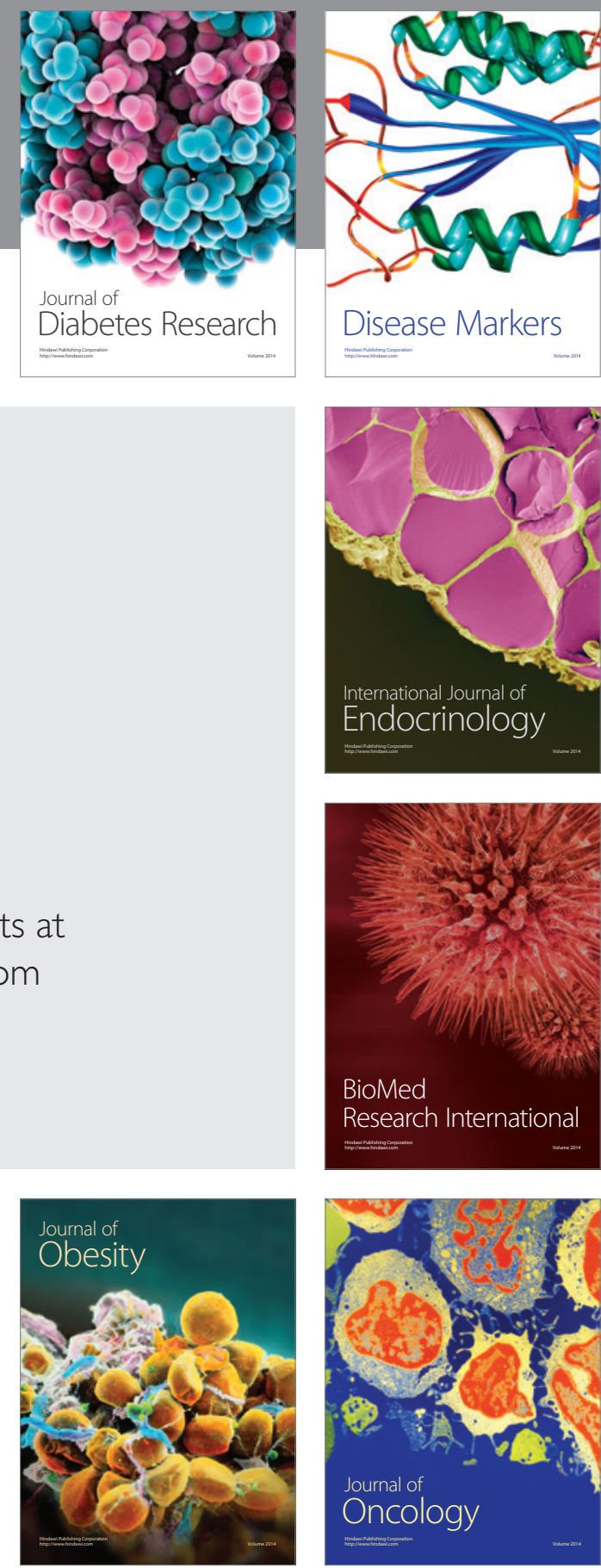

Disease Markers
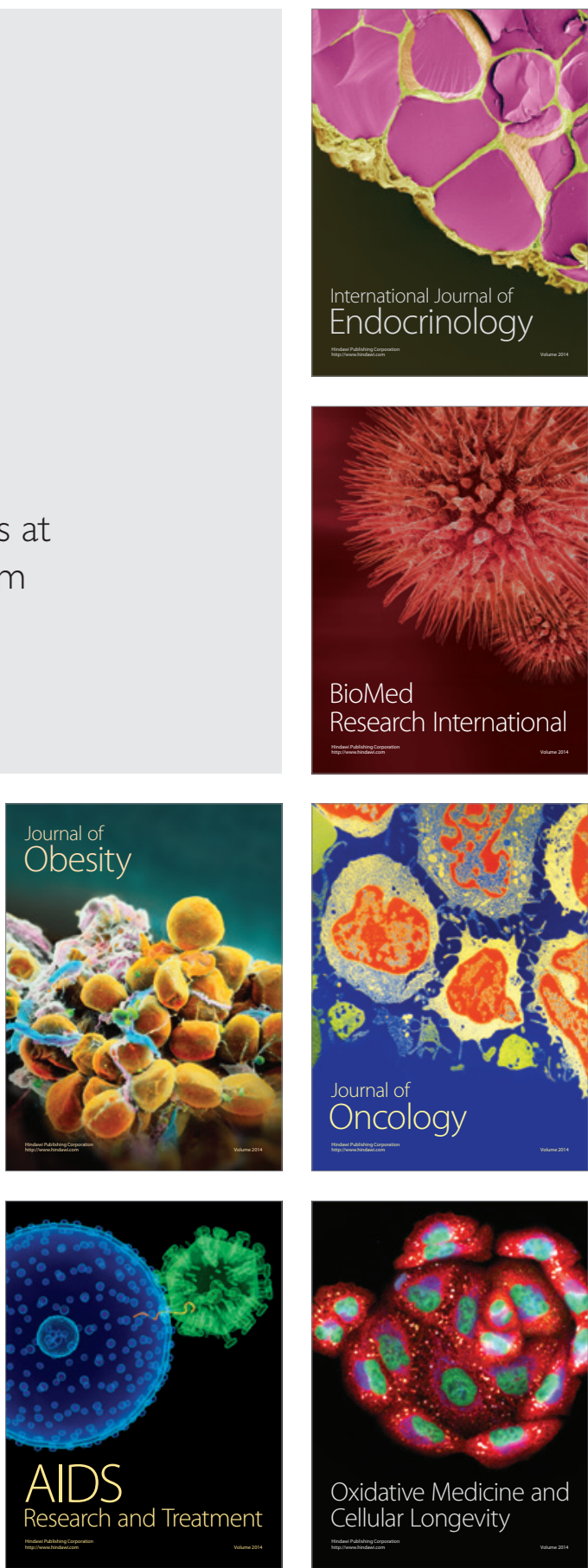